\title{
Cerebrospinal Fluid Rhinorrhea
}

National Cancer Institute

\section{Source}

National Cancer Institute. Cerebrospinal Fluid Rhinorrhea. NCI Thesaurus. Code C84627.

Discharge of cerebrospinal fluid through the nose. 